\title{
Electrocardiogram Domain
}

National Cancer Institute

\section{Source}

National Cancer Institute. Electrocardiogram Domain. NCI Thesaurus. Code C49626.

A subject domain utilized for the submission of information encompassing and representing data, vocabulary or records related to electrocardiogram. 